\title{
Attaque syllabique à deux éléments dans une société multiculturelle
}

\author{
Ataque silábico com dois elementos numa sociedade \\ multicultural
}

\author{
Teresinha de Moraes Brenner* \\ Universidade Federal de Santa Catarina
}

\begin{abstract}
The Portuguese syllabic system contains a consonantal onset with two elements $C_{2}$. The first one corresponds to an obstruent, plosive or fricative, and the second to a tap or a lateral. This paper presents an interesting configuration of the linguistic variational process related to the fishermen located in Florianópolis, S.C., Brazil. The multilinear phonology exposes the phenomenon under the universal principles and the local parameters, recovering an unified and satisfactory description. A scientific explanation of the phonological system, theme of this article, asks for an evaluation of the research and a revision in the educational area, including the Applied Linguistics.
\end{abstract}

\section{Keywords}

Linguistic variation; Syllabic attack, Onset and sonority, Phonological processes

\section{Resumé}

Le système syllabique du portugais comprend une attaque consonantique à deux éléments, $\mathrm{C}_{2}$. Le premier correspond à une obstruante, occlusive ou fricative, et le second, à un battement ou à une latérale. Ce cadre se comporte d'une façon très intéressante dans le processus de la variation linguistique chez 
les pêcheurs de Florianópolis, S.C., Brésil. La phonologie multilinéaire conçoit le phénomène sous la perspective des principes universels et des paramètres locaux capables d'attribuer aux faits une description unifiée et pertinente. Une explication plus scientifique des phénomènes de la langue et, en particulier, de la phonologie du portugais, thème de cet article, demande une évaluation de la recherche et une révision des sciences de l'éducation, incluant la Linguistique Appliquée.

\section{Mots-clés}

Variation linguistique; Attaque syllabique; Aperture et sonorité; Processus phonologiques 


\section{PROPOSITION}

ette étude porte sur la variation linguistique chez les pêcheurs de Florianópolis, S.C., Brésil. Leur grammaire, quant aux processus variationnels compris dans l'attaque syllabique à deux éléments, soutient des principes comparables à ceux de la structure linguistique des enfants en période de maternelle. L'analyse comporte une interface entre la phonologie multilinéaire et l'accoustique. On corrobore les faits par des sonagrammes configurés, dans les années 90, par l'auteur de ce travail dans L'Institut de Linguistique et Phonétique Générales et Appliquées de Paris III, SorbonneNouvelle. On a utilisé un ordinateur PC et le programme UNICE. Les données du corpus ont été récoltées dans les plages de Florianópolis.

La phonologie multilinéaire configure le mot ou la phrase en deux plans distincts où s'inscrivent trois lignes principales: celle de la syllabe, un squelette de positions pures ou vides de contenu phonétique ou phonologique, et celle des segments. La variation est conçue comme un phénomène associé au flottement d'éléments au niveau du lexique. On prédit comme flottant le second élément de l'attaque de la syllabe maximale du portugais $C^{2} V C^{2}$. Le premier $C$ correspond à une plosive [ \pm voisée] ou à une fricative [ \pm voisée]. Le second est rempli par un battement [+cor +ant] ou une latérale [+ cor, + ant]. Dans le phénomène variationnel, les éléments liquides sont remplaçables l'un par l'autre, ils peuvent s'effacer ou se déplacer et ils comportent encore d'autres modifications contextuelles.

Pour la description des processus phonologiques, on s'appuie sur une échelle de la sonorité proposée par Clements, 1988, et sur une autre avancée par Brenner, 1996, pour le portugais du Brésil. Dans le dernier cas, on se borne au cadre des liquides où s'intègrent le battement et la latérale, porteurs du degré maximal de sonorité et 
d'aperture par rapport à toutes les autres catégories consonantiques. On perçoit le battement comme plus fermé que la latérale, caractérisée par une structure presque formantique. Ainsi, si l'on se prend au schème $C C V$, le battement s'affaiblit-il dans la position de la latérale et ce dernier segment se renforce dans le contexte du premier.

La contribution des modèles multilinéaires se montre très intéressante pour résoudre les problèmes concernant la dscription de la variation chez les pêcheurs et les implications dans le cadre de l'apprentissage de la langue maternelle. L'étude scientifique du langage impose, en effet, une révision de la politique de l'éducation et, en particulier, le renouvellement de la formation professionnelle des enseignants de l'école élémentaire, secondaire et supérieure. Une connaissance plus approfondie de la langue leur permettra de mieux comprendre la grammaire standard et la variation linguistique afin de confronter les structures de différents dialectes sociaux et régionaux. Il leur sera, enfin, possible d'appliquer des méthodes adéquates et efficaces à l'enseignement.

\section{MODÈLE MULTILINÉAIRE: ATTAQUE SYLLABIQUE À DEUX ÉLÉMENTS}

Le modèle multilinéaire distribue les éléments du composant phonologique dans une configuration à trois couches principales: la syllabique, la squelettale et la segmentale. Les trois s'articulent avec le niveau prosodique et avec celui des traits phonologiques. Tous les niveaux se disposent en deux plans distincts séparés par la ligne centrale, le squelette, occupée par des positions vides de contenu phonétique ou phonologique. La ligne initiale syllabique métrique, binairement branchée en attaque et rime, s'insère, donc, dans le squelette, ainsi que la segmentale et celle des traits phonologiques. La configuration multilinéaire distribue les catégories et leurs éléments en couches distinctes et ils peuvent interagir entre eux sur le niveau qui leur appartient ou sur d'autres. 
La théorie multilinéaire tridimensionnelle conçoit encore une idée innovatrice, utilisée par Encrevé (1988) entre autres: le flottement. Selon cet auteur, tout élément est prévu potentiellement dans le lexique de la langue et il est passible de concrétisation ou non dans l'acte de la parole. La conception de choix des éléments virtuels dans le lexique permet l'organisation de grammaires particulières comprises dans un système linguistique plus large. Ainsi, le fottement fonde-t-il le concept de variation linguistique: les éléments flottants peuvent s'incorporer ou non aux différentes positions de la couche syllabique, segmentale, squelettale, prosodique ou à celle des traits phonologiques. En vue de description, le processus phonologique se rattache au flottement dans n'importe quel niveau d'une configuration géométrique.

Pour mieux expliquer l'organisation d'une grammaire dialectale comme celle des pêcheurs, on s'appuie sur Encrevé (1988), en spécial, qui propose comme d'autres phonologues, des principes et des contraintes universels associés à des paramètres locaux régissant un système spécifique. En somme, un modèle universel peut rendre compte d'un système linguistique comme celui du portugais et des grammaires dialectales que la langue recouvre.

Cet article se rapporte particulièrement au constituant attaque de la ligne syllabique. Il se compose, normalement, d'un seul élément consonantique, quand la rime contient une voyelle nucleus et facultativement une coda, consonne en finale de syllabe. La structure de base universelle de la syllabe équivaut à $C V$, étant néanmoins $C V C$ très fréquente dans les langues du monde.

Le portugais comporte une attaque et une coda à deux éléments, mais jamais les deux avec cet index. La syllabe maximale de cette langue se configure comme suit:

(1) $\mathrm{C}^{2} \mathrm{~V}^{*} \mathrm{C}^{2}$

Le symbole $V^{*}$ remplace l'index. En vérité, le portugais accepte trois vocoïdes au maximum, c'est-à-dire, une voyelle et deux semivoyelles. Quant à l'attaque à deux éléments, il se compose pareillement 
à celui de l'anglais où la première consonne est [-sonorante] et la seconde [+sonorante, -nasale] (CLEMENTS \& KEYSER, 1983, p. 42, apud Brenner 1996, p. 274). En portugais, le premier $C$ de l'attaque $C C$ se restreint aux obstruantes, représentées par l'occlusive, étant la fricative limitée à la labiale. Soient: [p], [b], [t], [d], [k], [g], [f], [v]. Le second $C$ équivaut aux liquides [1] et [r], latérale et battement. La formation des groupes $C C$ dans la langue en étude indique que [dl] se montre nul et que [vl] est très rare. On pose ci-dessous les séquences $C C$ du portugais:

(2) (a) [pr][pl][br][bl][tr][t]][dr]]kr][k]][gr][gl]

(b) [ff][fl][vr][vl]

Pour expliquer la structure des séquences formées d'une obstruante (occlusive ou fricative) et d'une liquide (latérale ou battement), on introduit ci-dessous le tableau proposé par Clements (1988), et repris par Milliken (1988) (BRENNER, 1996, p. 521) qui dispose toutes les catégories consonantiques d'après la sonorité et l'aperture. Elles sont regroupées selon les traits de classe majeure:

$$
\begin{array}{llllll}
\mathrm{O} & \mathrm{N} & \mathrm{L} & \mathrm{G} & \mathrm{V} & \\
\hline- & - & - & - & + & \text { [ouvert] } \\
- & - & - & + & + & \text { [vocoïde] } \\
- & - & + & + & + & \text { [approximant] } \\
- & + & + & + & + & \text { [sonorant] } \\
\hline
\end{array}
$$

$\begin{array}{llllll}0 & 1 & 2 & 3 & 4 & \text { Échelle de sonorité }\end{array}$

Dans le cas d'une attaque à deux éléments en portugais, le premier, une occlusive ou une fricative, comprend le degré zéro de sonorité et il se caractérise comme [-ouvert]. Le second, une liquide, approximante, porte le degré 2 de sonorité et il est aussi [- ouvert].

Une étude à propos de l'aperture des phonèmes du portugais faite par Mattoso Camara Jr. (1980) attribue le degré 0 aux occlusives, 
1 aux fricatives, 2 aux nasales, 3 aux liquides, 4 aux semi-voyelles et 5 aux voyelles. Si l'on compare les deux échelles, on voit que tout élément consonantique de la première est toujours [-ouvert], quand la seconde distribue l'aperture selon le décroissement de la constriction dans le canal buccal. Ces données se montrent très pertinentes, vu qu'elles accusent la liquide, second élément de l'attaque, comme plus sonore, plus ouverte que les autres consonnes et, par conséquent, comme plus favorable aux processus de la variation linguistique.

Le cadre des liquides du portugais comprend trois souscatégories: la vibrante, le battement et la latérale. Inspirée de Clements, Brenner (1996, p. 557), a proposé un tableau distribuant les liquides selon l'aperture et la sonorité sur les axes du mode et du lieu d'articulation, en conformité avec le schème syllabique. Quant au mode d'articulation, la vibrante, la liquide plus fermée, a été signalée par le degré 1 , le battement, 2 et la latérale, 3. La première peut se modifier en fricative, rangeant plusieurs variantes dans cette catégorie. La première et surtout la dernière présentent des variantes dans le système des semi-voyelles. Ce travail concerne celles qui portent le degré 2 et 3, le battement et la latérale. On présente cidessous un tableau appuyé sur Brenner (1996, p. 629) comprenant leur organisation:

(4) MODE

LIEU

\begin{tabular}{cccc}
2 & 1 & 2 & 3 \\
BAT & & $\mathrm{C} / \mathbf{r} / \mathrm{V}$ & $\mathrm{V} / \mathbf{r} / \mathrm{V}$ \\
3 & & & \\
LAT & $/ \mathrm{l} / \mathrm{V}$ & $\mathrm{C} / \mathrm{l} / \mathrm{V}$ & $\mathrm{V} / \mathrm{l} / \mathrm{V}$ \\
\hline
\end{tabular}

Le tableau (4) se rapporte aux constituants compatibles avec la position de second élément consonantique de l'attaque, les segments porteurs des traits [+cor, +ant], le battement et la latérale. Par conséquent, la latérale palatale / $\mathrm{\Lambda}$ / et la vélaire / $\mathbf{t}$ / sont exclues. Clements (1993) regroupe les consonnes et les voyelles quant au lieu 
d'articulation en quatre catégories: [labial], [coronal], [dorsal] et [pharyngal]. La [coronal] comporte [ \pm antérieur].

La position de coda du portugais est restreinte à la latérale [dorsale, vélaire], à la vibrante, à la fricative [coronale, \pm antérieure] et à la nasale. Cette information est pertinente pour la compréhension da la métathèse qui permet la réalisaton du second segment de CC comme coda syllabique.

Le lieu d'articulation, schématisé dans le tableau (4), prédit que la position 1 de l'axe horizontal n'est jamais occupée par le battement. En portugais, ce segment est toujours intervocalique. Par contre, la latérale [coronale] apparaît en début de syllabe initiale de mot. Pour cette analyse, seule la position 2 est pertinente: battement et latérale intégrés en $\mathrm{C}^{2}$. Le cadre (5) réunit des informatons contenues dans (3) et (4):

(5) Attaque $C C$

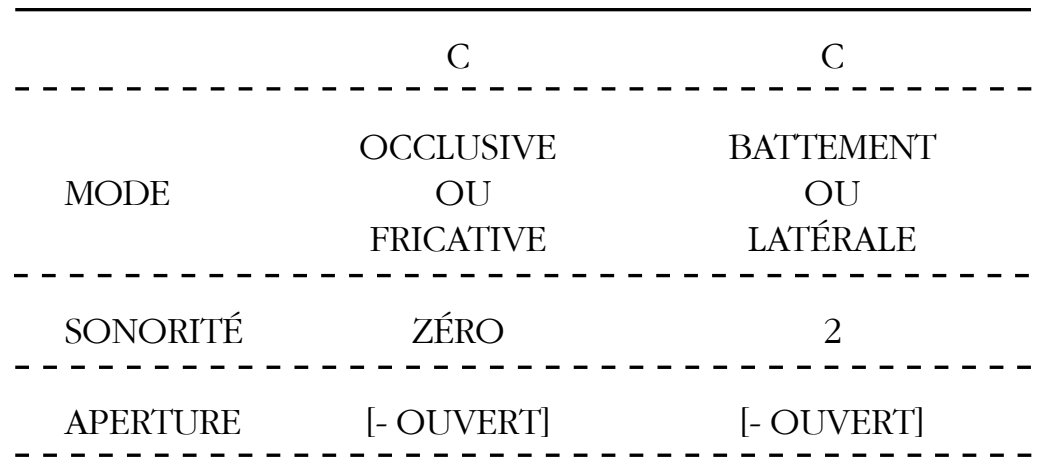

À propos de la co-articulation, le battement dans la structure $C C V$ assimile les valeurs de la voyelle qui le suit plutôt que celles de la consonne qui le précède. Il y a, donc, plus de sonorité dans le schéme $[\beta \mathrm{P}]$ que dans $[\pi \mathrm{P}]$. Dans la latérale le phénomène préserve les mêmes critères, mais la consonne maintient ses valeurs et ses courbes formantiques plus stables. 


\section{VARIATION: ATTAQUE $C_{2}$}

On a déjà mentionné ci-dessus que les processus plus importants de l'attaque syllabique dans la séquence CC se rapportent au second élément, plus sonore, plus ouvert, rapproché des voyelles, et, par conséquent, plus transparent à la variation. Il arrive, donc, que le premier se fait très rarement connu dans le phénomène variationnel. Normalement, le battement est affaibli, par substitution, dans la position de la latérale, la latérale est renforcée dans la position du battement. Les deux segments de $\mathrm{C}_{2}$ sont encore suscetibles d'éffacement. La syllabe peut recevoir un autre élément par ajout. Si le battement change de place dans la syllabe, il acquiert un autre statut phonémique. Les processus se distribuent, donc, en cinq, se distinguant les deux premiers: affaiblissement, renforcement, ellipse, épenthèse et métathèse.

Le second élément de l'attaque en CCV se comporte, donc, comme un élément flottant dans le lexique des pêcheurs, en particulier, de la couche pauvre en général et dans le langage enfantin; il peut subir de différentes réalisations contextuelles. Ce flottement n'existe, cependant, pas dans le portugais standard, où les groupes se maintiennent presque stables sous une seule forme de base abstraite et dans la structure de surface.

\subsection{Affaiblissement}

Le remplacement de / $/$ [ +cor +ant] par /1/ [+cor +ant] dans le schème $C C V$ provient de la possibilité d'un choix entre les deux segments réalisé au niveau du lexique. Le fait s'explique généralement par la substitution dans l'axe vertical qui peut être associée ou non à l'assimilation ou à la dissimilation entre les éléments contextuels.

Peu de données sont suffisantes pour la comprobation du processus. Soient les deux formes parallèles: 
(6) Forme dialectale Portugais standard Traduction

(a) ['klonike $]$ ['kronike $] \quad$ chronique

(b) [Re'klute] [Re'krute] recrue

(c) [a'glariw $]$ [a'grarib $] \quad$ agraire

(d) [es'klupulu] [es'krupulu] scrupule

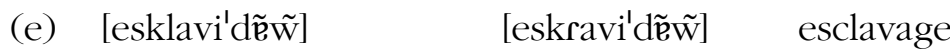

(f) [a'kliliku] [a'kriliku] fibre synthétique

On illustre l'assimilation vérifiée en (6)(d) à travers la configuration hiérarchique (7). La liquide de la syllabe tonique d'un proparoxyton est remplacée par celle de la posttonique finale. Le schème vocalique comprend trois fois la voyelle [u] à partir de la syllabe tonique, de telle forme que la structure CV finale s'inclue dans la syllabe accentuée. La distribution de l'accent suit les critères déjà posés ci-dessus (MATTOSO CAMARA Jr., 1980; 1972, apud BRENNER, 1996) pour le portugais: syllabe tonique, 3 ; syllabe prétonique 1 et posttonique, 0 . Suit la configuration:

(7) [es'krupulu]

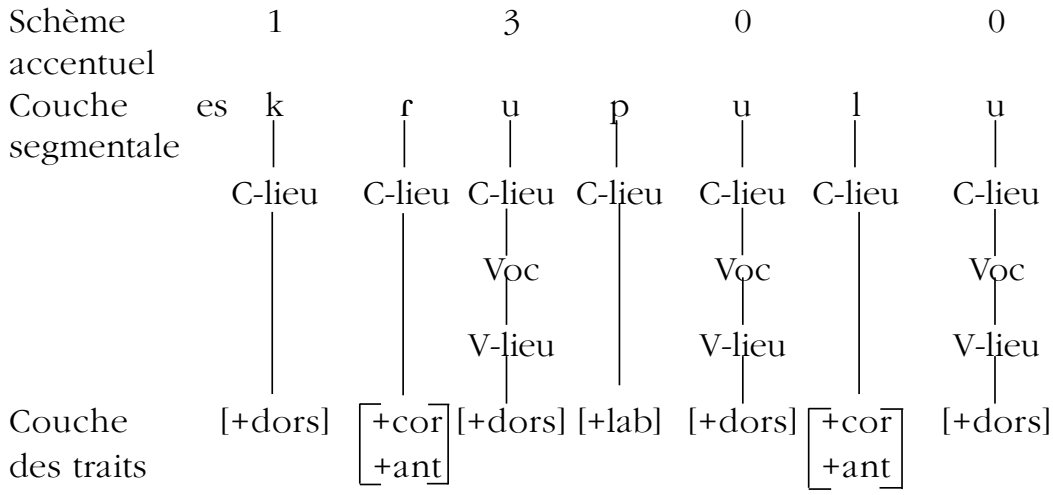

Le battement assimile en (7) le mode d'articulation de [1] d'où résulte $(6)(d)$ : la latéralité se répand à gauche. Soit: 
(8) Assimilation de la latéralité:

L'assimilation de /1/

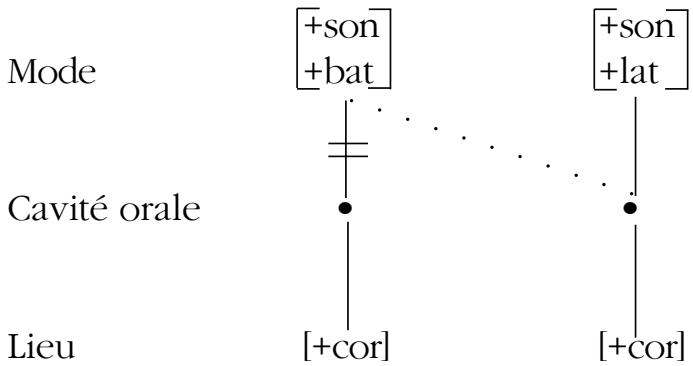

On peut expliquer autrement: l'affaiblissement du battement est favorisé par la présence de la latérale en syllabe finale posttonique.

La configuration (7) présente, selon une prédiction de la théorie des traits phonologiques, une barrière à l'assimilation proposée en (8). Une condition de blocage interdit le croisement des traits d'association sur un même plan (GOLDSMITH, 1976; CLEMENTS, 1985 apud CLEMENTS, 1993, p. 130):

(9) Contrainte universelle de non-croisement

Les traits d'association ne peuvent pas se croiser sur un même plan.

La configuration (7) montre que le [p] [consonne plosive] représente une barrière à l'assimlation de la latéralité proposée en (8). Le processus s'organise sur le plan du mode d'articulation composé par une occlusive+battement, une occlusive et une latérale. Il faut, donc, justifier la transparence de l'occlusive labiale qui est normalement opaque aux croisement des lignes d'association.

Il semble prudente une description du mot phonologique "escrúpulo" dans une configuration tridimensionnelle globale où interfèrent tous les plans - surtout celui de la prosodie. La latérale s'insère dans une syllabe atone finale de mot proparoxyton. Elle est la plus sonore et la plus ouverte dans le cadre des consonnes se comportant comme élément flottant. Par contre, le [p] [labial], dans la première posttonique d'un proparoxyton, se situe dans un contexte 
de labialité vocalique (trois [u]). Le point d'articulation le rapproche, donc, des voyelles. On sait que la voyelle est presque toujours transparente aux processus.

L'encadrement dans un contexte [labial] de nature vocalique ainsi que son insertion dans une position syllabique faible (accent zéro) permet d'envisager l'occlusive comme un élément incapable d'empêcher l'assimilation de la latéralité de droite à gauche. Cette lecture de (7) s'explique comme possible ou probable ayant la valeur d'introduire la discussion ce qui permet l'inclusion d'autres arguments ou d'autres propositions.

Le sonagramme qui suit illustre la dissimilation en tant qu'affaiblissement du battement:

(10) [Re'forma'glarye] (réforme agraire)

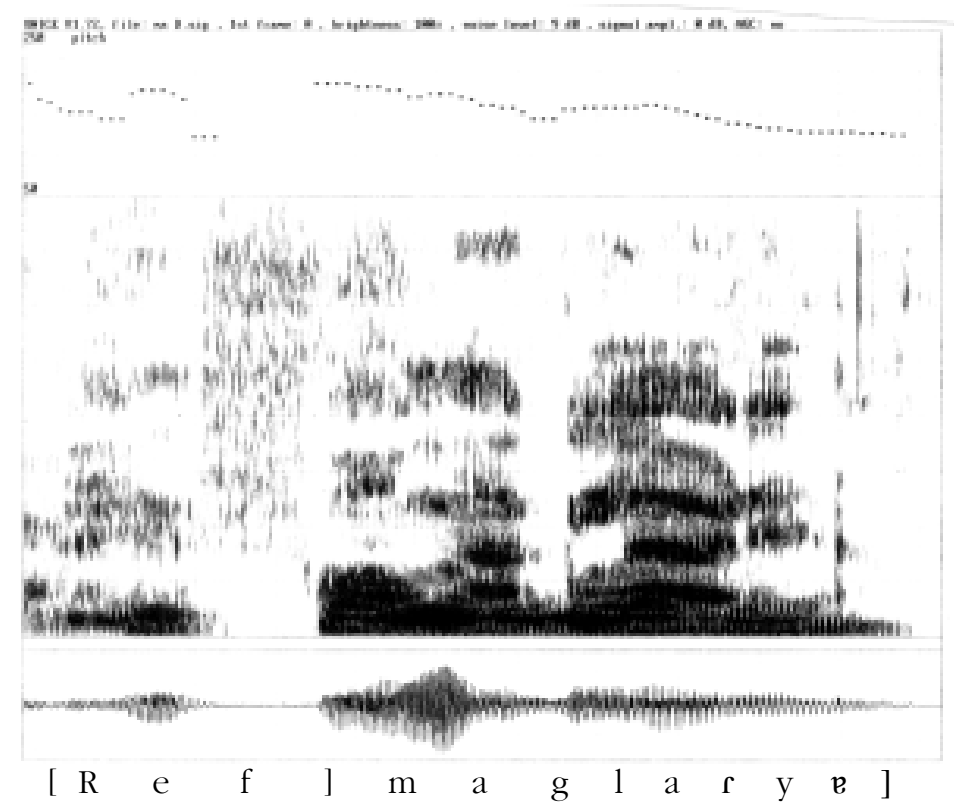

On perçoit en (10) que la phrase contient deux vibrantes dans les deux premières syllabes et un battement dans la derrière - deux phonèmes apparentés par sa nature. Les informateurs utilisaient 
surtout la vibrante et rarement la variante fricative. Le [u] fort du Latin a dérivé la vibrante et le battement du Portugais. La suite de deux consonnes $C C$ d'une syllabe porteuse de l'accent principal faculte que le battement de la structure de base soit affaibli par dissimilation. Il est remplacé, donc, par la liquide qui comporte des traits de lieu identiques [+cor,+ant], la latérale. Le sonagramme n'accuse qu'un seul battement inséré au début de la sylllabe finale de la phrase. La présence de [1] formantique est nettement perçue dans la séquence [gl] tout à fait différente de l'organisation de [r] qui subit l'influence de [a], à gauche et de [y], à droite.

On a étudié jusqu'ici le schème [OCCLUSIVE + BATTEMENT]. On trouve peu de données concernant le système [FRICATIVE + BATTEMENT] où le second élément est affaibli en latérale, étant promu dans l'échelle de l'aperture et de la sonorité. Suivent des exemples:

(11) Forme dialectale Portugais standard Traduction
(a) ['flet $\left.\int \mathrm{i}\right]$
['fret $\mathrm{i}$ i]
Fret
(b) [es'flege]
[es'frege]
V. frotter

On est parvenu à la constatation que le schème $C C V$ comprenant au début une occlusive est plus fréquent que celui portant une fricative comme premier élément. L'occlusive représente la consonne la plus fermée, la moins sonore, d'oú dérive probablement sa combinaison plus libre avec les éléments de l'autre extrémité du cadre consonantique, les liquides. De ce fait, s'explique la réduction de données concernant la fricative dans le processus variationnel illustré en (11).

\subsection{Renforcement}

La latérale après l'occlusive et la fricative comporte mieux l'opération relative à son renforcement en battement que le battement accepte son affaiblissement en latérale. Le phénomène n'est pas observé dans le portugais standard. Le processus est très répandu dans la couche pauvre. Suivent quelques données: 
(12) Forme dialectale Portugais standard Traduction Avec l'occlusive
(a) $\left[\mathrm{e}^{\mathrm{l}} \mathrm{ze} \mathrm{m} \mathrm{pru}\right]$
[e' $\left.\mathrm{ze}^{\mathrm{m}} \mathrm{plu}\right]$
exemple
(b) [a'krare]
[a'klare]
V. éclairer
(c) ['prebi]
['plebi]
plèbe
(d) [dekri'now]
[dekli'now]
V. décliner
(e) ['prake]
['plake]
plaque
Avec la fricative
(f) [fro'reste]
[flo'reste]
forêt
(g) [fre'sade]
[fle'sade]
(h) ['frame]
['flame]
coup de flèche
(i) [a'fritu]
[a'flitu]
flamme
(j) [fru'tue]
[flu'tue]
affligé
V. flotter

Suit le sonagramme:

(13) ['prene] (pleine)

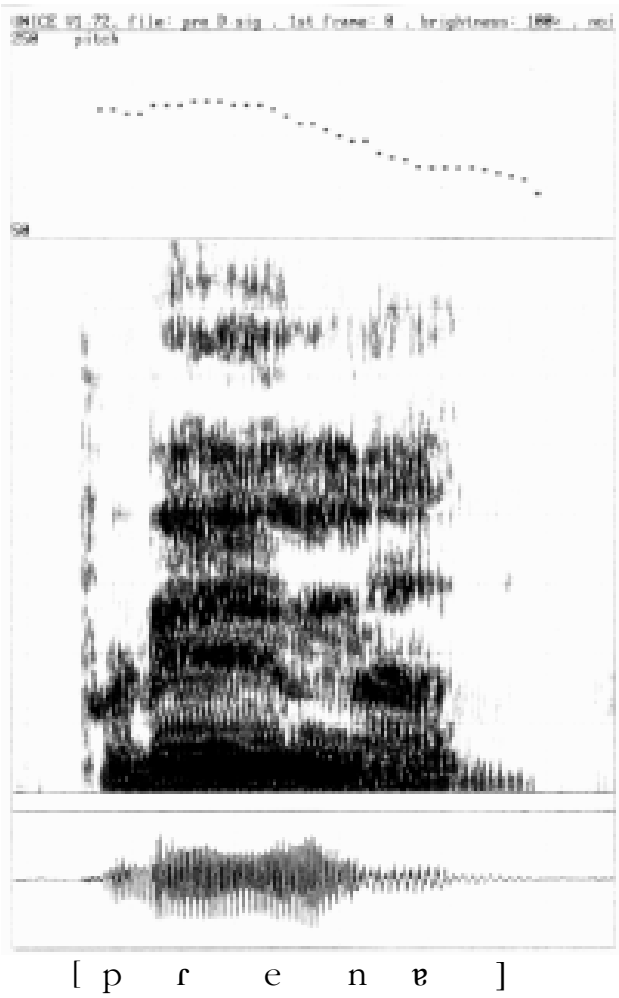


Le sonagramme montre qu'il y a un battement et non une latérale formantique suivant le [p] initial de mot. On reprend ce qu'on a déjà affirmé ci-dessus: la substitution de la latérale par le battement est très fréquente dans le dialecte des pêcheurs et dans la couche pauvre. Le processus est aussi enregistré dans le langage enfantin comprenant tant l'occlusive que la fricative. Le lexique de l'enfant diffère, néanmoins, de celui des pêcheurs et l'acquisition des phonèmes suit des stades bien définis. Le procesus articulatoire de l'homme voué à la pêche tend, par contre, à la fermeture, fait culturellement expliqué. Les deux grammaires maintiennent, cependant, des rapports. L'analyse permet ainsi qu'on pose le paramètre suivant:

(14) La variation, par renforcement, est, en CC, plus vérifiable chez l'élément plus ouvert de l'échelle, la liquide latérale, qui occupe, en conséquence, la position d'un segment plus fermépar le remplacement.

\subsection{Ellipse}

L'ellipse est corroborée par nombre de données trouvées surtout dans la couche pauvre. À titre d'illustration, on sélectionne les suivantes:

(15) Forme dialectale
(a) [a'pume]
(b) [is'kevu]
(c) [po'bleme]
(d) [es'kotu]
(e) [Re'peze]

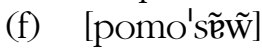
(g) [a'pesu]

Portugais standard

[a'prume]

[es'krevu]

[pro'bleme]

[es'krotu]

[Re'preze]

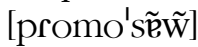

[a'presu]
Traduction

V. se redresser

V. écrire

problème

scrotum

éclouse

promotion

estime

La configuration phonologique de l'ellipse se présente comme suit:

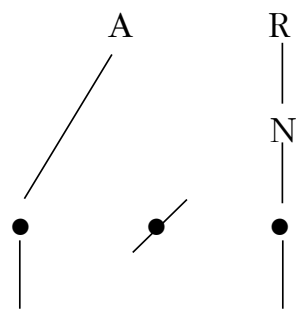


Le second élément de l'attaque ne s'associe pas au squelette. La position vide résulte du flottement compris dans le lexique.

Le sonagramme illustre l'ellipse:

(17) [iske'vewe'klonike] (il a écrit la chronique)

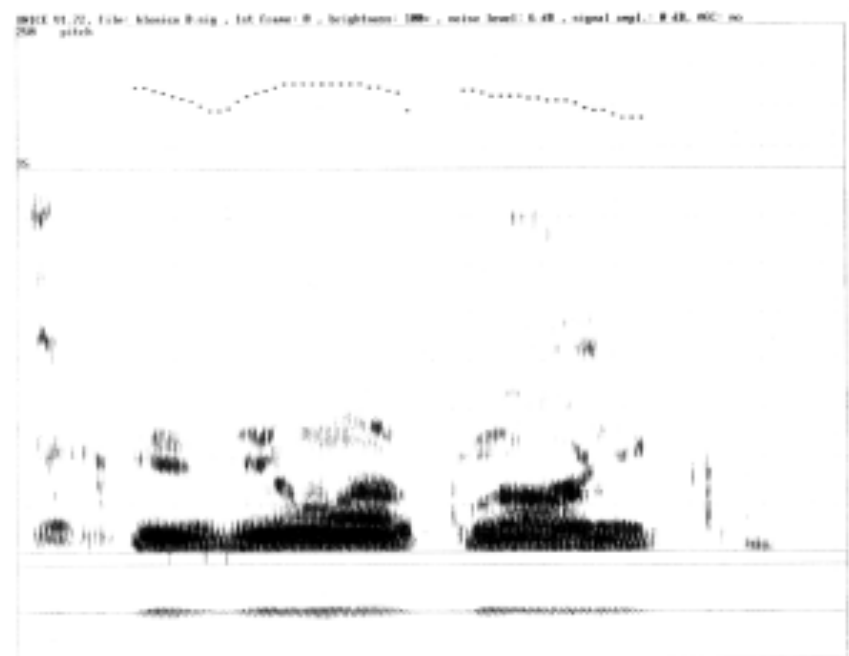

$\left[\begin{array}{llllllllllllllll}\mathrm{i} & \mathrm{s} & \mathrm{k} & \mathrm{e} & \mathrm{v} & \mathrm{e} & \mathrm{w} & \mathrm{e} & \mathrm{k} & \mathrm{l} & \mathrm{o} & \mathrm{n} & \mathrm{i} & \mathrm{k} & \mathrm{e} & \end{array}\right]$

On a montré l'ellipse du battement précédé d'une occlusive en (15), de même que la substitution de cet élément par la latérale dans $C C$ du dernier mot de la phrase. Le processus s'observe également après la fricative, comme en ['fute] qui remet, dans le registre standard, à ['frute] (fruit). La latérale suivant l'occlusive s'efface normalement, de même qu'il arrive quand elle correspond au second $C$ en $C C$, étant le premier une fricative. On rappelle, cependant, que le lexique avec la fricative est beaucoup plus restreint dans la langue. Le phénomène le plus frappant reliant la fricative et le battement se rapporte à l'ellipse.

L'effacement du second élément $C C V$, schème marqué, s'explique aisément chez les enfants et dans les cultures populaires par la recherche de la forme non-marquée, plus simple et naturelle dans la langue parlée, $C V$. 


\subsection{Métathèse}

Le battement, en portugais, est issu, diachroniquement, de la lénition d'une vibrante intervocalique du Latin. Le deux segments, vibrante et battement, sont, alors, apparentés. De ce fait, on peut expliquer une métathèse attestée chez les couches populaires: dans le mot "prateleira", par exemple, le battement, second élément en $C C$, se déplace et il se réalise comme une vibrante insérée dans la position de coda de la syllabe initiale. Autrement dit, le second segment de l'attaque perd son statut de battement et, en s'associant à la position de coda, il se concrétise comme une vibrante, compatible avec le nouveau shème- $C V C$.

(18) Forme dialectale Portugais standard Traduction [prate'leyre] [par̃teleyre] étagère

Suit la figure:

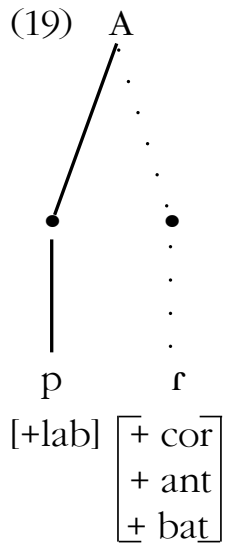

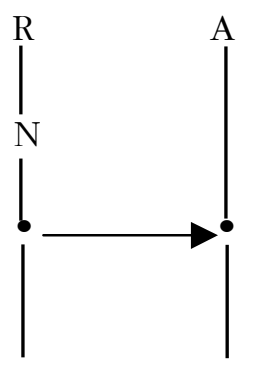

$\mathrm{p}$

[+lab]

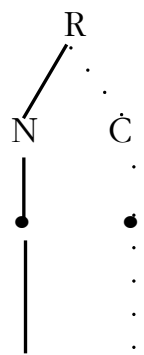

a

$\left.\begin{array}{c}\tilde{r} \\ + \text { cor } \\ + \text { ant } \\ + \text { vib }\end{array}\right]$

On présente le sonagramme 
(20) [parrte'leyre'nove] (étagère neuve)

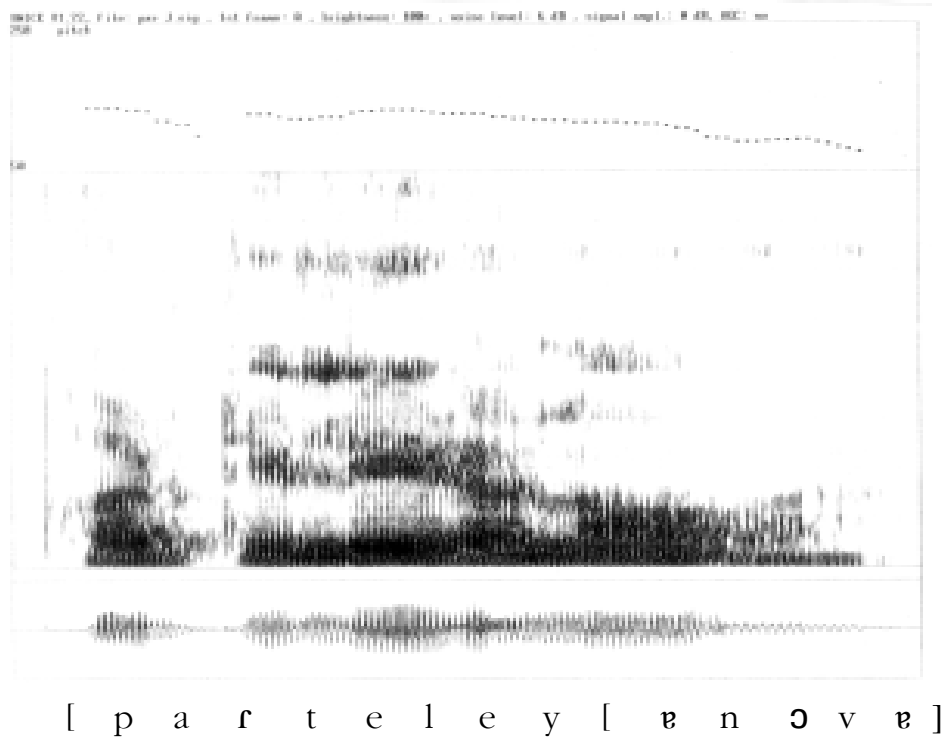

Le sonagramme montre une syllabe initiale formée par une occlusive, une voyelle ouverte suivies d'une coda vibrante. On peut mentionner d'autres cas de métathèses:

(21) Forme dialectale Portugais standard Traduction

(a) ['plori] ['proli] progéniture

(b) [be'flave $] \quad$ [ble'fave $] \quad$ v. dujer

(c) [at'flitu] [a'flitu] affligé

En (a), on vérifie que, dans la forme dialectale, si l'on prend pour base la structure standard du portugais, le second élément de $C C V$ occupe la position de $C$ dans la dernière syllabe et que le phonème accroché à $C$ de celle-ci s'insère dans la première. On atteste, donc, une permutation entre les liquides dans un mot à deux syllabes, $C C V$ et $C V$. En (b), la latérale de $C C V$ de la première syllabe s'intègre dans la seconde syllabe: le schème $C C$ se déplace de la première vers la 
seconde syllabe. En (c), la latérale [+cor], de la seconde syllabe, élément ${ }^{2}$ de $C C$, se réalise comme coda [+dors] de la première. Les phénomènes mettent toujours en jeu la syllabe tonique. Dans le dialecte des pêcheurs, on trouve bien d'autres exemples de métathèse.

\section{5. Épenthése}

La vraie épenthèse est un peu plus rare: l'ajout de toute une colonne flottante dans la configuration phonologique. Soient:

(22) Forme dialectale Portugais standard Traduction

(a) [Ro'brow] [Ro'bow $\quad$ v. voler

(b) [Refres'telu] [Refes'telu] rengorgement

(c) ['plublike $\quad$ ['publike $]$ publique

Les trois cas illustrés s'expliquent par l'assimilation. Les deux premiers présentent la vibrante initiale de syllabe qui favorise l'insertion de la liquide apparentée, un battement, dans la seconde syllabe, découlant le schème CC. En (c), CC se refait dans la prétonique avec la copie de la latérale de la syllabe tonique.

Pour conclure, on expose les paramètres qui régissent la grammaire du dialecte des pêcheurs de Florianópolis:

(23) Paramètres locaux

(a) d'après le modèle multilinéaire, on intreprète le second élément de l'attaque du schème $C C V$ comme flottant dans la structure de base;

(b) le segment flottant en $C C$ correspond normalement à une liquide, un battement ou une latérale $[+\mathrm{COR},+\mathrm{ANT}]$, étant le premier $C$ correlé à une occlusive ou à une fricative;

(c) dans l'ensemble, le lexique des pêcheurs s'organise autour des éléments plus fermés du cadre consonantique, étant l'occlusive plus choisie dans l'attaque $C_{2}$;

(d) le lexique de la langue est plus restreint pour la fricative dans le schème $C C$; 
(e) le processus plus frappant dans la séquence fricative + battement est l'ellipse du dernier élément;

(f) la variation se montre plus large et variée dans les séquences CC avec l'occlusive, degré " 0 " dans l'échelle de la sonorité;

(g) les échelles d'aperture et de sonorité rendent compte de l'affaiblissement du battement et du renforcement de la latérale de l'attaque $C_{2}$;

(h) le flottement est plus répandu, quand le second $C$ de l'attaque, dans le processus de substitution, et dans le cadre variationnel, en général, est une latérale, élément plus sonore et plus ouvert de l'échelle de sonorité et d'aperture et, par conséquent, plus transparent;

(i) l'effacement du second $C$ par les couches populaires équivaut, dans la recherche de la forme non-marquée, à la normalisation de la syllabe métrique.

\section{VARIATION: INTERFACE ENTRE SCIENCE ET ÉDUCATION}

La variation linguistique dans une société multiculturelle entraîne des problèmes et des difficultés dans la politique éducationnelle. Si on préconise l'enseignement de la langue officielle dans l'école, le système éducationnel doit accorder un traitement spécial aux différents dialectes régionaux et sociaux. Il faut, par conséquent, insérer et articuler les recherches des sciences du langage dans un système politique plus complexe. La sociolinguistique et la psycholinguistique, par exemple, peuvent envisager avec plus de précision les structures culturelles et linguistiques des couches régionales et sociales diversifiées.

Dans ce travail, on accuse l'importance de la phonologie, en particulier, et les implications générales de la sociolinguistique, de la psycholinguistique et de la linguistique appliquée, pour l'enseignement de la langue maternelle. La recherche phonologique démontre un comportement différent de l'attaque syllabique à deux éléments, si 
l'on met en confrontation le dialecte des pêcheurs et le parler des enfants d'un côté et de l'autre, le portugais standard. Le langage enfantin corrélé au phénomène de l'acquisition du langage s'oppose à son tour à celui des dialectes régionaux et sociaux au niveau du lexique. Une description scientifique des faits selon la phonologie multilinéaire permet d'interpréter le phénomène d'une façon unitaire dans la langue, malgré la diversification.

L'enfant, au moment de l'entrée à l'école, organise la grammaire en conformité avec le stade psycholinguistique de son âge et d'après la culture régionale et sociale. L'imposition du niveau standard de la langue par l'école peut déclencher chez lui une confrontation entre son niveau linguistique et celui du système officiel. Il se fait, alors, nécessaire, du point de vue des professionnels, une connaissance plus scientifique et plus approfondie de la structure de la langue standard et de ses dialectes. Il s'impose, par conséquent, une politique de l'éducation adéquate.

Recherche et enseignement doivent marcher ensemble. Il en résulte l'exigence d'un renouvellement de la formation professionnelle traditionnelle des enseignants de l'école maternelle, de l'école élémentaire, secondaire et supérieure. La contribution scientifique leur permettra de mieux comprendre les phénomènes de la variation et le fonctionnement du système linguistique dans son ensemble.

Selon l'exposé ci-dessus, le modèle multilinéaire se montre capable de décrire l'organisation métrique de la syllabe et le comportement des éléments de l'attaque $C C V$ dans la langue portugaise. La variation linguistique suit des paramètres très précis et réguliers. Il faut les comprendre pour interpréter la grammaire individuelle et régionale des élèves afin de diriger d'une façon adéquate le processus de l'enseignement/apprentissage. La linguistique appliquée subsiste dans une interdisciplinarité comptant sur les fondements de différents champs de la linguistique, des sciences sociales et de la pédagogie en articulation avec l'informatique, la philosophie et la politique. 


\section{NOTE}

* Cet article a été présenté sous forme de communication dans le Colloque AFLA 2000, organisé par l'Association Française de Linguistique Aplliquée, Paris, et il a été révisé, en 2005, et encore en 2006, en vue de parution.

\section{BIBIOGRAPHIE}

BRENNER, Teresinha de Moraes. Une approche de la latérale du portugais du Brésil parlé par les pêcheurs de Florianópolis (S.C.): conséquences pour l'enseignement/ apprentissage de la langue maternelle. In: COSCAS \& SCLIARCABRAL, Linx, Paris, Université Paris X, 29 (2): p. 99-110, 1993.

BRENNER, Teresinha de Moraes. Une approche multilinéaire de la variation dialectale des consonnes occlusives et liquides chez les pêcheurs de Florianópolis, S.C., Brésil. 1996. Thèse (Doctorat) - Sorbonne-Nouvelle, Paris.

BRENNER, Teresinha de Moraes. La linguistique appliquée aux manuels d'alphabétisation. International review of education. Hamburg, UNESCO Institute for Education, 45 (2): p. 215-35, 1999.

CAMARA JR., Joaquim Mattoso. Princípios de lingüistica geral. 6. ed. Rio de Janeiro: Padrão, 1980.

CLEMENTS, George. Lieu d'articulation des consonnes et des voyelles. In: LAKS \& RIALLAND. Architecture des représentations phonologiques. Paris: CNRS Éditions, 1993.

CLEMENTS \& HUME, E. The internal organisation of speech sounds. In: GOLDSMITH, John. The handbook of phonological theory. Cambridge: B. Blackwell, 1995.

ENCREVÉ, Pierre. La liaison avec et sans enchaînement. Paris: Éditions du Seuil, 1988. 